\title{
Altered spontaneous brain activity patterns in patients with corneal ulcer using amplitude of low-frequency fluctuation: An fMRI study
}

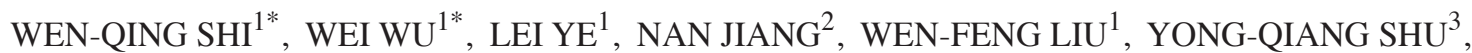 \\ TING SU ${ }^{2}$, QI LIN ${ }^{1}$, YOU-LAN MIN ${ }^{1}$, BIAO LI ${ }^{1}$, PEI-WEN ZHU ${ }^{1}$ and YI SHAO \\ ${ }^{1}$ Department of Ophthalmology, The First Affiliated Hospital of Nanchang University, \\ Jiangxi Province Ocular Disease Clinical Research Center, Nanchang, Jiangxi 330006; \\ ${ }^{2}$ Fujian Provincial Key Laboratory of Ophthalmology and Visual Science, Eye Institute of Xiamen University, \\ Xiamen, Fujian 361102; ${ }^{3}$ Department of Radiology, The First Affiliated Hospital of Nanchang University, \\ Jiangxi Province Medical Imaging Research Institute, Nanchang, Jiangxi 330006, P.R. China
}

Received September 19, 2018; Accepted March 18, 2019

DOI: $10.3892 / \mathrm{etm} .2019 .7550$

\begin{abstract}
The aim of the present study was to investigate the altered spontaneous brain activity in patients with corneal ulcer (CU) through the amplitude of low-frequency fluctuation (ALFF) technique and the association with their visual performance. A total of 40 patients with CU and 40 healthy controls (HCs) matched for sex, age and educational level were enrolled. Resting-state functional magnetic resonance imaging (rs-fMRI) was performed to examine the probands. Spontaneous cerebral activity variations were investigated using the ALFF technique. The average ALFF values of the $\mathrm{CU}$ patients and the $\mathrm{HCs}$ were classified by utilizing receiver operating characteristic (ROC) curves. Contrary to HCs, the CU patients had significantly lower ALFF values in the left cerebellar anterior lobe, right middle frontal gyrus and left middle frontal gyrus, but higher ALFF values in the right cerebellar inferior lobe, left cerebellar inferior lobe, left inferior temporal gyrus, right fusiform gyrus, left superior frontal gyrus, right angular gyrus and bilateral superior frontal gyrus. ROC curve analysis of each brain region indicated that the accuracy of ALFF value specificity between the CU and $\mathrm{HCs}$ of the area under the curve was perfect. In conclusion, abnormal spontaneous activities were detected in numerous brain regions of $\mathrm{CU}$ patients, which may provide useful
\end{abstract}

Correspondence to: Professor Yi Shao, Department of Ophthalmology, The First Affiliated Hospital of Nanchang University, Jiangxi Province Ocular Disease Clinical Research Center, 17 Yong WaiZheng Street, DongHu, Nanchang, Jiangxi 330006, P.R. China E-mail: freebee99@163.com

*Contributed equally

Key words: ALFF, fMRI, corneal ulcer, resting state, spontaneous activity information for understanding the dysfunction of CU. These activity changes in brain regions may be used as effective clinical indicators for CU.

\section{Introduction}

Corneal ulcer (CU) is a common ophthalmological disease and is one of the prime causes of monocular blindness worldwide, particularly in developing countries (1). CU, including infectious and non-infectious $\mathrm{CU}$, is the prime cause of corneal opacity and the fourth largest cause of blindness in the world (2). The clinical manifestations include blurred vision, pain, photophobia and lacrimation, as well as obvious visual impairment $(3,4)$.

Ophthalmic examination may indicate disappearance of the corneal luster, a decrease in transparency or ulcer formation (5). Severe cases may cause corneal perforation, intraocular infection or even blindness (6).

Functional magnetic resonance imaging (fMRI) is a method for detecting brain changes $(7,8)$, and may be utilized to enhance the current knowledge on ophthalmological diseases. fMRI is a neuroimaging research technique for exploring the brain and central nervous system; it may be used for monitoring the spontaneous neuronal activity of the human brain and provide novel explanations for the pathophysiology of certain conditions (9). It is suitable for the study of central mechanisms, since it does not require any radioactive tracers, and it may detect and accurately locate spontaneous neuronal activity in the human brain by combining functional with structural imaging (10). Amplitude of low-frequency fluctuation (ALFF) is one of the useful resting-state fMRI analytic methods for assessing the activity of brain regions during rest (11). Studies have demonstrated that the ALFF performs well based on its test-retest reliability $(9,12)$. The ordinary calculation and reliable feature of the ALFF measurement make it an appropriate and helpful tool for analyzing resting-state fMRI data of disease characteristics. In previous studies by our group, the ALFF method has been successfully 
used to assess the neurological status in certain eye diseases, including optic neuritis (13), glaucoma (14) and comitant strabismus (15). ALFF is considered a reliable and sensitive measurement that may be applied to accurately assess spontaneous neural activity.

The aim of the present study was to investigate the altered spontaneous brain activity and its association with the behavioral performance in CU patients by using ALFF technology and provide a potential neural mechanism underlying the pathogenesis of $\mathrm{CU}$.

\section{Patients and methods}

Subjects. A total of $40 \mathrm{CU}$ patients examined at the First Affiliated Hospital of Nanchang University (Nanchang, China) between April 2016 and June 2017 were enrolled in the present study.

The diagnostic criteria for $\mathrm{CU}$ were as follows: i) A history of corneal trauma; ii) corneal infiltration, turbidity, local tarnish, tissue defects and mixed congestion; iii) symptoms of irritation, including increased sensitivity to light, lacrimation decreased vision and eye secretions, and iv) anterior chamber empyema in the patients with severe disease.

The inclusion criteria for CU patients was as follows: i) A duration of $\mathrm{CU}$ of at least 14 days, ii) no abnormality of brain parenchyma on craniocerebral MRI, iii) right-handedness, iv) no foreign implant in the body and v) fMRI was performed prior to CU surgery. The exclusion criteria were as follows: i) Long-term medical treatment for blindness, ii) a history of surgery in bilateral eyes, iii) bilateral late blindness, iv) bilateral congenital blindness and v) psychiatric disorders (including bipolar disorder, depression and sleep disorder) and vi) cerebral infarction-associated diseases (e.g., cerebral infarction, cerebral hemorrhage or cerebral vascular malformations). A total of 40 healthy control subjects (HCs) that were matched with the corresponding CU group in terms of age, sex and education status were also recruited. All of the participants fulfilled the following criteria: i) MRI findings without apparent deformities in the brain parenchyma; ii) no psychiatric disease, cerebral infarction or cardiovascular disease; iii) no drugs or alcohol addiction; iv) capable of undergoing MRI examination.

The present study was in accordance with the Declaration of Helsinki and was formally approved by the Medical Ethics Committee of the First Affiliated Hospital of Nanchang University. All volunteers provided written informed consent.

MRI parameters. MRI was performed using a Trio 3-Tesla MR scanner (Siemens AG, Munich, Germany). All of the subjects were instructed to keep their eyes closed while awake and maintain natural breathing during the scan (14). A 3-dimensional spoiled gradient recalled-echo pulse sequence was applied to acquire the functional data with the parameters set as follows: Acquisition matrix, 256x256; field of view, 250x250 mm; echo time, 2.26 msex; repetition time, 1,900 msec; thickness, $1.0 \mathrm{~mm}$; gap, $0.5 \mathrm{~mm}$; flip angle, $9^{\circ}$ ). A total of 240 functional images (acquisition matrix, 64x64; field of view, $220 \times 220 \mathrm{~mm}$; thickness, $4.0 \mathrm{~mm}$; gap, $1.2 \mathrm{~mm}$; repetition time, $2,000 \mathrm{msec}$; echo time, $30 \mathrm{msec}$; flip angle, $90^{\circ}$; axial views, 29. A total of 176 structural images were thereby obtained and the examination lasted for $15 \mathrm{~min}$.

fMRI data analysis. Functional data analysis was performed using a method described in a previous study by our group (15). First, MRIcro software (Nottingham University) was applied to remove any incomplete data. During magnetization equilibration, the first 15 time-points were discarded. Data Processing Assistant for advanced edition of resting-state (rs)-fMRI software (DPARSFA 4.0; http://rfmri.org/DPARSF) was used for head motion correction, spatial normalization, slice timing, data conversion into the digital imaging communications in medicine format, and full-width smoothing with a Gaussian kernel of $6 \times 6 \times 6 \mathrm{~mm}^{3}$ at half-maximum, based on the rs-fMRI data analysis toolkit (REST; http://www.restfmri. net) and Statistical Parametric Mapping software (SPM; http://www.fil.ion.ucl.ac.uk/spm). Subjects were excluded if they had 1.5 angular motion or the maximum offset of $x, y$ or $\mathrm{z}$ direction exceeded $1.5 \mathrm{~mm}$ during the fMRI examination. The number of patients in the CU group and the $\mathrm{HC}$ group was originally 46. After removal of certain patients due to head motion and the consequent removal of respective control individuals to maintain equal group sizes, 40 patients remained in each group. Although it is not a one-to-one correspondence, it has consulted a statistical expert to indicate that it has no effect on the study. The Friston six head-motion parameters were used to regress out head-motion effects based on previous literature that revealed that higher-order models were more effective $(16,17)$. Furthermore, linear regression was applied to remove other various sources of spurious covariates and their temporal derivatives, including the signal from a ventricular region of interest and a region centered in the white matter (18). It is worth noting that in the data of the present study, the global signal did not shrink as was the case in previous studies by our group $(9,19,20)$, which may be due to the debated elimination of global signals during the resting-state data pre-processing $(21,22)$. After the head motion correction, the fMRI images were normalized to the space standard of the Montreal Institute of Neurological Institute (23) using a standard echo plane imaging template, and at the same time, the images were resampled with a resolution of $3 \times 3 \times 3 \mathrm{~mm}$. After pre-treatment, the time series of each voxel was linearly decreased in order to reduce the low frequency drift, heart noise and physiological high-frequency respiration, along with linear detrending of the time series. In order to reduce the global impact of variability across the participants, the ALFF of each voxel was divided by the global mean ALFF value for each participant.

Statistical analysis. Values are expressed as the mean \pm standard deviation. Variables of the demographic and clinical features of the $\mathrm{CU}$ and $\mathrm{HC}$ groups were compared using SPSS software version 20.0 (IBM Corp., Armonk, NY, USA) via an independent-samples t-test. The statistical threshold of the voxel level for multiple comparisons according to the Gaussian random field (GRF) theory was set at a level of $\mathrm{P}<0.05$. Furthermore, AlphaSim was corrected at a cluster size of $>40$ voxels and a significance level of $P<0.01$. All ALFF maps were z-transformed with Fisher's r-to-z transformation to reduce the influence of individual variation for group statistical 
Table I. Characteristics of participants included in the study.

\begin{tabular}{lcccc}
\hline Parameter & CU group (n=40) & HCs (n=40) & t & P-value \\
\hline Males/females & $26 / 14$ & $26 / 14$ & N/A & $>0.99$ \\
Age (years) & $51.25 \pm 5.46$ & $51.98 \pm 5.18$ & 0.251 & 0.824 \\
Body weight (kg) & $63.12 \pm 7.35$ & $63.89 \pm 6.73$ & 0.181 & 0.892 \\
Right-handedness (n) & 40 & 40 & N/A & $>0.99$ \\
Duration of CU (days) & $25.75 \pm 5.65$ & N/A & N/A & N/A \\
\hline
\end{tabular}

Sex was analyzed using the Chi-square test, while age and weight were analyzed using independent t-tests. HCs, healthy controls; N/A, not applicable; CU, corneal ulcer.

Table II. Brain areas with significantly different ALFF values between groups.

\begin{tabular}{|c|c|c|c|c|c|c|c|c|c|}
\hline \multirow[b]{2}{*}{ Comparison of ALFF } & \multirow[b]{2}{*}{$\mathrm{L} / \mathrm{R}$} & \multirow[b]{2}{*}{ Brain region } & \multicolumn{3}{|c|}{ MNI coordinates } & \multirow[b]{2}{*}{$\mathrm{BA}$} & \multirow[b]{2}{*}{ Peak voxels } & \multirow[b]{2}{*}{ T-value } & \multirow[b]{2}{*}{ P-values } \\
\hline & & & $\mathrm{X}$ & $\mathrm{Y}$ & $\mathrm{Z}$ & & & & \\
\hline \multicolumn{10}{|l|}{$\mathrm{UDs}<\mathrm{HCs}$} \\
\hline 1 & $\mathrm{~L}$ & Cerebellar anterior lobe & -36 & -51 & -27 & l & 61 & -5.35 & $\mathrm{P}<0.001$ \\
\hline 2 & $\mathrm{R}$ & Middle frontal gyrus & 30 & -6 & 54 & 6 & 19 & -4.5773 & $\mathrm{P}<0.001$ \\
\hline 3 & $\mathrm{~L}$ & Middle frontal gyrus & -24 & -9 & 51 & 6 & 18 & -4.1103 & $\mathrm{P}<0.001$ \\
\hline \multicolumn{10}{|l|}{ UDs $>\mathrm{HCs}$} \\
\hline 1 & $\mathrm{R}$ & Cerebellar inferior lobe & 21 & -69 & -60 & l & 94 & 5.557 & $\mathrm{P}<0.001$ \\
\hline 2 & $\mathrm{~L}$ & Cerebellar inferior lobe & -36 & -72 & -57 & l & 218 & 5.786 & $\mathrm{P}<0.001$ \\
\hline 3 & $\mathrm{~L}$ & Inferior temporal gyrus & -57 & -48 & -27 & 20 & 24 & 4.4602 & $\mathrm{P}<0.001$ \\
\hline 4 & $\mathrm{R}$ & Fusiform gyrus & 51 & -36 & -27 & 18 & 16 & 4.4716 & $\mathrm{P}<0.001$ \\
\hline 5 & $\mathrm{~L}$ & Superior frontal gyrus & -18 & 57 & 3 & 9 & 25 & 4.8905 & $\mathrm{P}<0.001$ \\
\hline 6 & $\mathrm{R}$ & Angular gyrus & 48 & -63 & 39 & 39 & 16 & 5.2397 & $\mathrm{P}<0.001$ \\
\hline 7 & $\mathrm{~B}$ & Superior frontal gyrus & 0 & -21 & 78 & 6 & 132 & 4.8908 & $\mathrm{P}<0.001$ \\
\hline
\end{tabular}

An independent-samples t-test was applied to obtain P-values for comparisons between CUs and HCs. ALFF, amplitude of low-frequency fluctuation; BA, Brodmann area; CU, corneal ulcer; HCs, healthy controls; MNI, Montreal Neurological Institute; L, left; R, right; B, bilateral; /, brain areas have no clear BA division.

comparisons and the independent t-test was used to compare group differences in the zALFF maps using the GRF method to correct for multiple comparisons and regressed covariates of age and sex with Statistical Parametric Mapping 12 software (The MathWorks, Inc.; two-tailed, voxel-level P<0.01, GRF correction, cluster-level $\mathrm{P}<0.05)$. The regions of the cerebrum with a distinctly different mean ALFF value between the CU subjects and $\mathrm{HCs}$ were analyzed using receiver operating characteristic (ROC) curves.

\section{Results}

Demographics and disease characteristics. No statistically significant differences were present between the $\mathrm{HC}$ and $\mathrm{CU}$ groups in body weight $(\mathrm{P}=0.892)$ or age $(\mathrm{P}=0.824)$, as indicated in Table I. The mean duration of $\mathrm{CU}$ was 25.75 \pm 5.65 days (Table I).

Differences in ALFF. In contrast to those of HCs, ALFF values of the CU patients were significantly lower in the left cerebellar anterior lobe, right middle frontal gyrus and left middle frontal gyrus, but higher in the right cerebellar inferior lobe, left cerebellar inferior lobe, left inferior temporal gyrus, right fusiform gyrus, left superior frontal gyrus, right angular gyrus and bilateral superior frontal gyrus (Figs. 1 and 2; Table II).

ROC curve analysis. The mean ALFF values of different brain regions were analyzed using the ROC curve method. The area under the ROC curve (AUC) represented the diagnosis rate. The AUC of ALFF values in different brain regions was as follows: i) CUs $>\mathrm{HCs}$ (Fig. 3A): Right cerebellar inferior lobe, 0.760 ( $\mathrm{P}<0.001)$; left cerebellar inferior lobe, $0.828(\mathrm{P}<0.001)$; left inferior temporal gyrus, $0.754(\mathrm{P}<0.001)$; right fusiform gyrus, $0.771 \quad(\mathrm{P}<0.001)$; left superior frontal gyrus, 0.812 $(\mathrm{P}<0.001)$; right angular gyrus, $0.807(\mathrm{P}<0.001)$; bilateral superior frontal gyrus, 0.785 ( $\mathrm{P}<0.001)$; ii) $\mathrm{CUs}<\mathrm{HCs}$ (Fig. 3B): Left cerebellar anterior lobe, $0.848(\mathrm{P}<0.001)$; right middle frontal gyrus, $0.778(\mathrm{P}<0.001)$; and left middle frontal gyrus, $0.773(\mathrm{P}<0.001)$. 

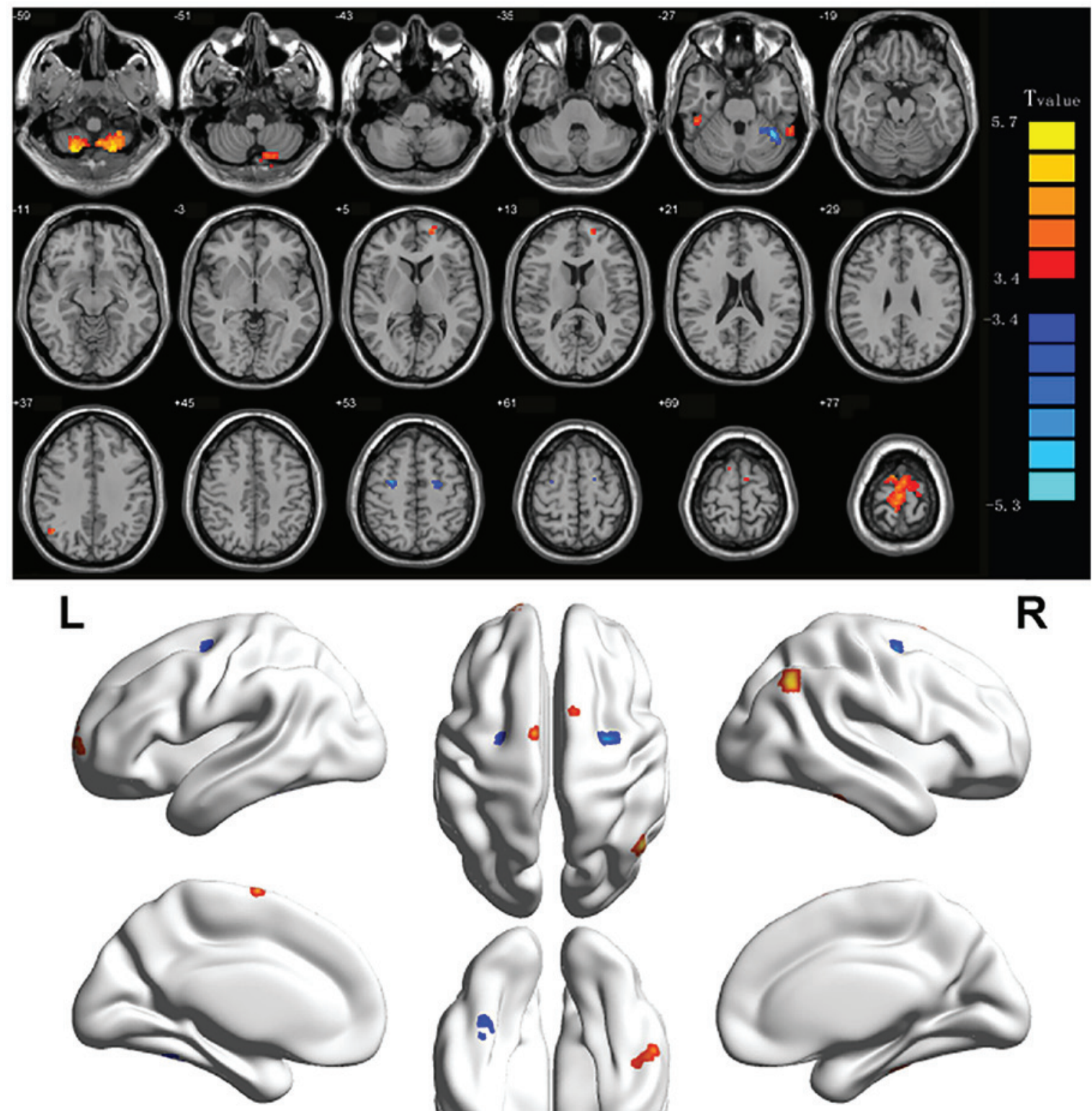

$\mathbf{R}$
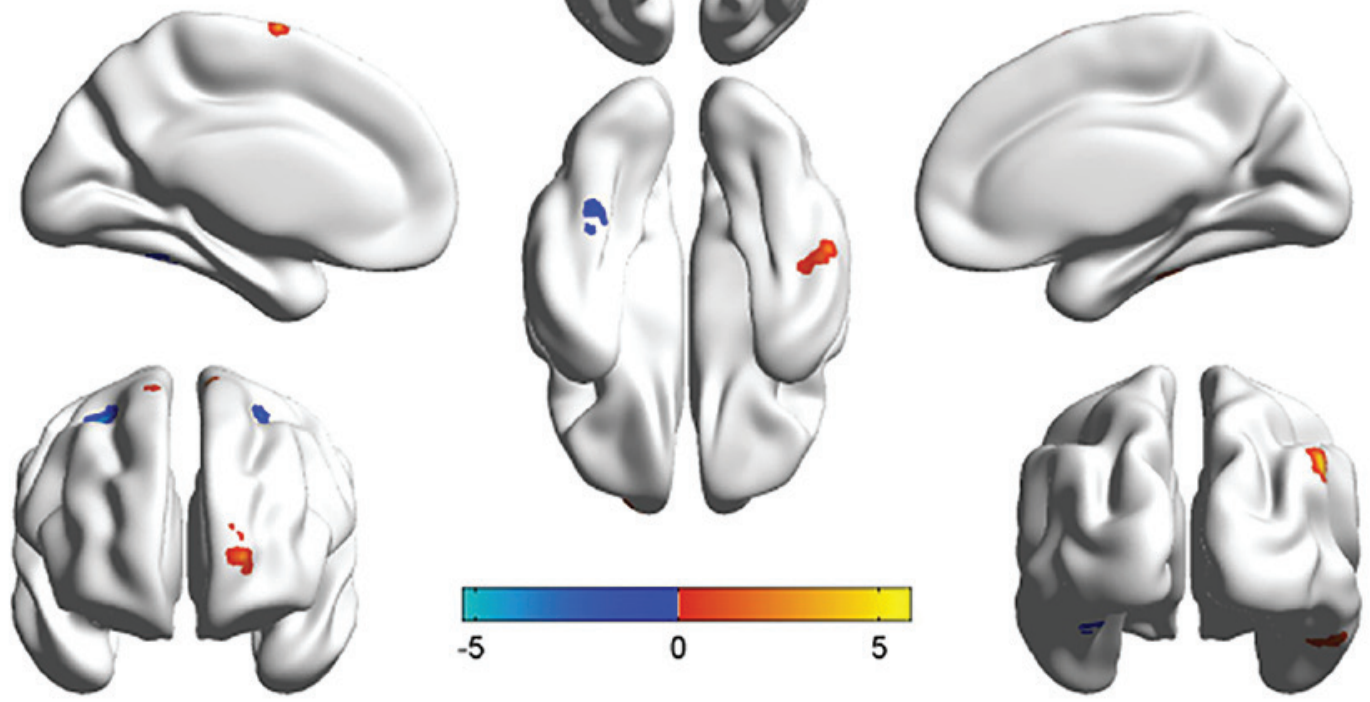

Figure 1. Significant differences in spontaneous brain activity between the CU group and HCs. Differences were observed in the left cerebellum anterior lobe, right middle frontal gyrus, left middle frontal gyrus, right cerebellar inferior lobe, left cerebellar inferior lobe, left inferior temporal gyrus, right fusiform gyrus, left superior frontal gyrus, right angular gyrus and bilateral superior frontal gyrus. The red areas denote brain regions with higher ALFF and the blue areas denote brain regions with lower ALFF $[\mathrm{P}<0.001$ for multiple comparisons using Gaussian random field theory $(\mathrm{z}>2.3 ; \mathrm{P}<0.001 ;$ cluster, $>13$ voxels; Alphasim corrected)]. ALFF, amplitude of low-frequency fluctuation; HCs, healthy controls; L, left; R, right; CU, corneal ulcer.

\section{Discussion}

The middle frontal gyrus (Fig. 4), which is involved in attention control (24) and working memory (25), comprises one-third of the frontal lobe. Chronic CU patients, appear to have difficulty avoiding inattention due to the impaired vision. Certain previous studies have indicated that depressed individuals had dysfunction in the middle frontal gyrus $(26,27)$. Visual impairment may directly affect physical function and performance through decreased ability to see where to walk and visualize the location of objects in space (28). Low vision has been associated with increased disability. Bruce and Hoff (29) indicated that disability may lead to depression. In support of these results, the present study indicated a decrease of 


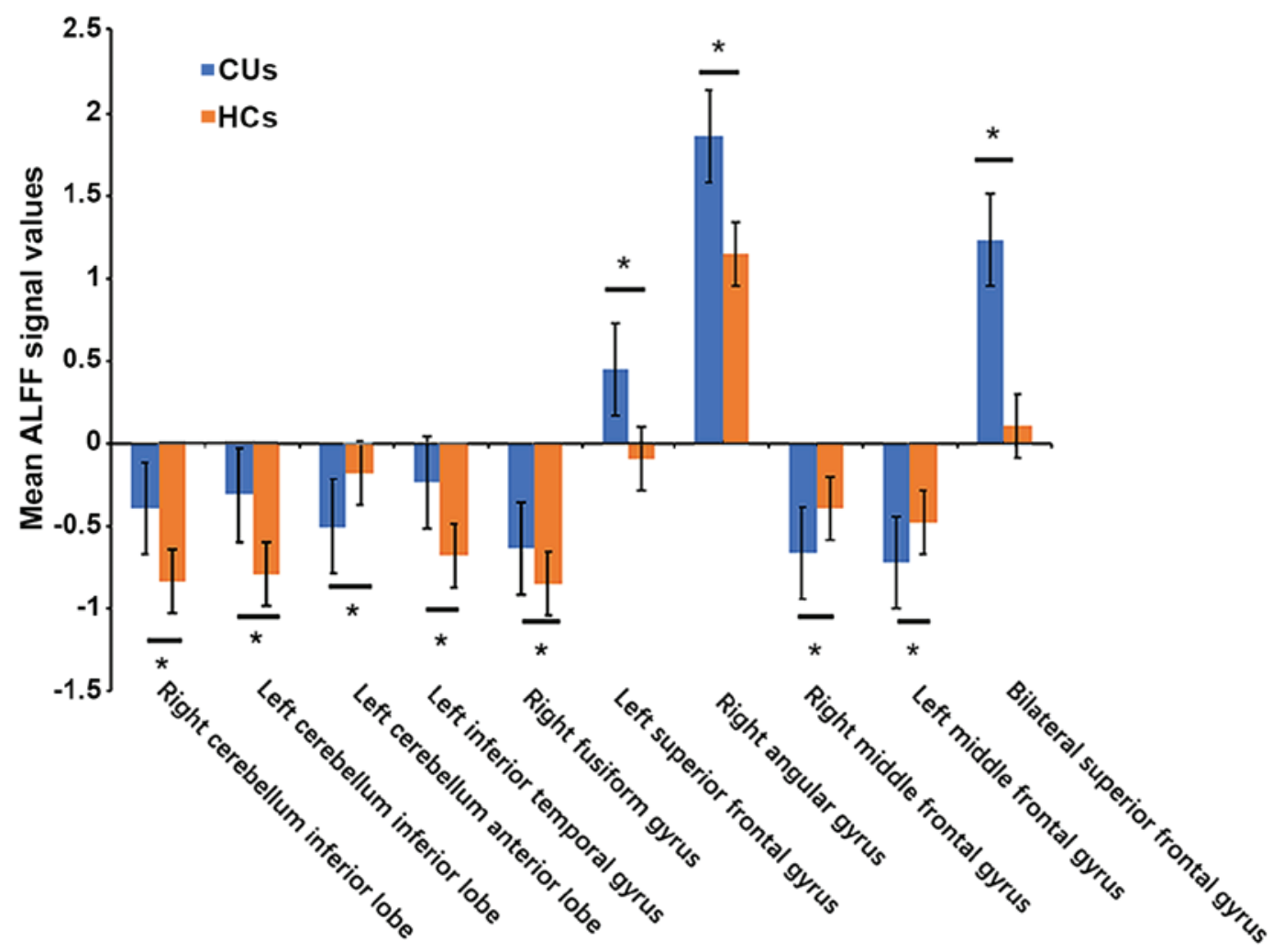

Figure 2. Means of altered spontaneous brain activity compared between the CU group and HCs. The statistical threshold was set at voxel with $\mathrm{P}<0.01$ for multiple comparisons using family-wise error correction ( $>2.3$; $\mathrm{P}<0.01$; cluster, $>40$ voxels). "P<0.05. HCs, healthy controls; $\mathrm{CU}$, corneal ulcer; ALFF, amplitude of low-frequency fluctuation; RCIL, right cerebellum inferior lobe; LCIL, left cerebellum inferior lobe; LITG, left inferior temporal gyrus; RFG, right fusiform gyrus; LSFG, left superior frontal gyrus; RAG, right angular gyrus; BSFG, bilateral superior frontal gyrus; LCAL, left cerebellum anterior lobe; RMFG, right middle frontal gyrus; LMFG, left middle frontal gyrus.

A

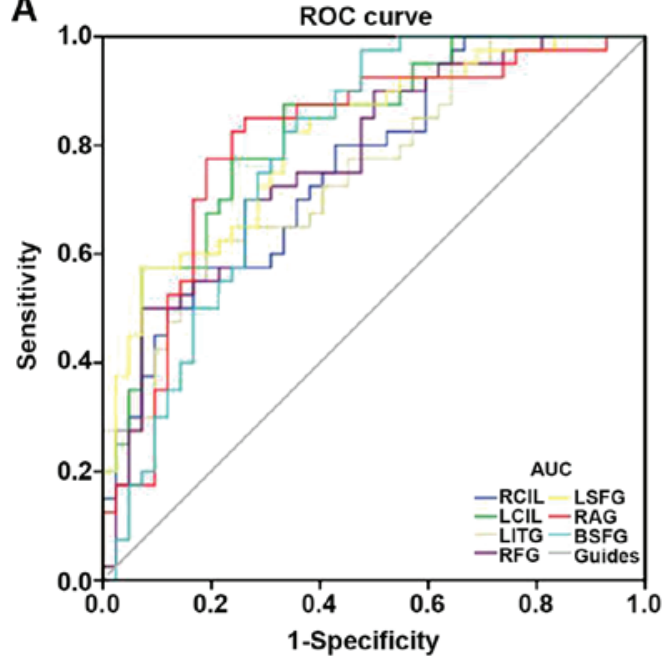

B

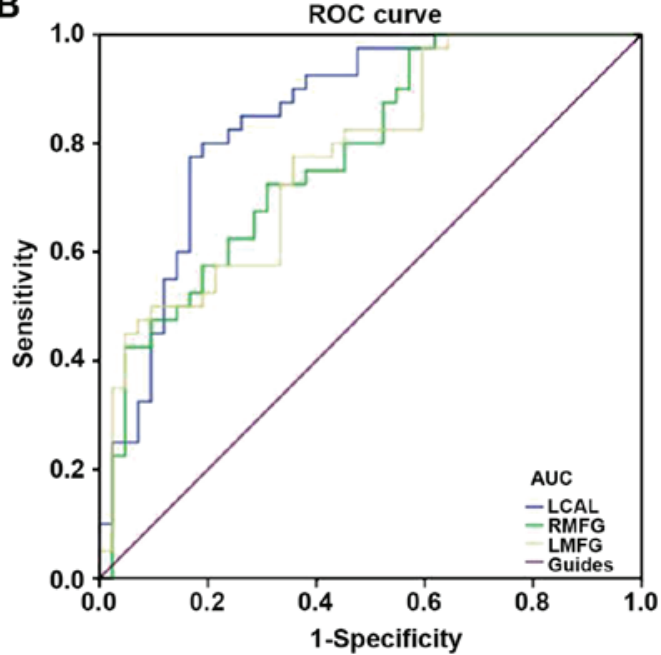

Figure 3. ROC curve analysis of the mean ALFF values for altered brain regions. (A) The area under the ROC curve for the RCIL was 0.760 (P $<0.001 ; 95 \%$ CI, $0.650-0.862)$, that for the LCIL was $0.828(\mathrm{P}<0.001 ; 95 \% \mathrm{C}$,: 0.741-0.915), that for the LITG was $0.754(\mathrm{P}<0.001 ; 95 \%$ CI, $0.651-0.857)$, that for the RFG was 0.771 ( $\mathrm{P}<0.001 ; 95 \% \mathrm{CI}, 0.670-0.872)$, that for the LSFG was 0.812 ( $\mathrm{P}<0.001 ; 95 \% \mathrm{CI}, 0.721-0.904)$, that for the RAG was 0.807 (P<0.001; 95\% CI, 0.708-0.906) and that for the BSFG was 0.785 ( $\mathrm{P}<0.001 ; 95 \% \mathrm{CI}, 0.684-0.885)$ [CUs $>\mathrm{HCs}$ ]. (B) The area under the ROC curve was 0.848 (P<0.001; 95\% CI, 0.763-0.933) for the LCAL, while that for the RMFG was 0.778 ( $\mathrm{P}<0.001$; 95\% CI, 0.679-0.877) and that for the LMFG 0.773 (P<0.001; 95\% CI, 0.674-0.873) [CUs<HCs]. ALFF, amplitude of low-frequency fluctuation; HCs, healthy controls; CU, corneal ulcer; ROC, receiver operating characteristic; RCIL, right cerebellar inferior lobe; LCIL, left cerebellar inferior lobe; LITG, left inferior temporal gyrus; RFG, right fusiform gyrus; LSFG, left superior frontal gyrus; RAG, right angular gyrus; BSFG, bilateral superior frontal gyrus; LCAL, left cerebellar anterior lobe; RMFG, right middle frontal gyrus; LMFG, left middle frontal gyrus.

the ALFF value in the left/right middle frontal gyrus of $\mathrm{CU}$ patients, which may also be associated with depression. The depression caused by $\mathrm{CU}$ might result in middle frontal gyrus dysfunction. 
The anterior lobe is a part of the cerebellum that mediates unconscious proprioception. The present study demonstrated that the ALFF value in the left anterior cerebellar lobe in the CU group was significantly reduced.

Traditionally, the function of the cerebellum is considered to be movement coordination (30). However, with the development and application of neuroimaging technology in recent years, the understanding on the role of cerebellum, particularly the cerebellar inferior lobe, has expanded to include emotional processing (31). Previous studies have indicated that patients with social anxiety examined by positron emission tomography had abnormal signals in the cerebellum, which was characterized by increased cerebral blood flow and led to the conclusion that the cerebellum is associated with anxiety (32). This is in line with the present results. Therefore, the high ALFF value of the cerebellar inferior lobe may be the result of anxiety in patients.

The inferior temporal gyrus (ITG), located on the lateral and inferior surface of the temporal neocortex, may be regarded as the language formulation central area and tertiary visual association cortex. Its functions include visual perception, cognitive language and memory (33). ITG is an important area of association that promotes cognitive processing and regulation of emotions. In addition, compared with $\mathrm{HCs}$, patients with somatoform pain disorder exhibit increased activation of the temporal region, suggesting that the temporal region activity may be associated with physical pain (34).

The fusiform gyrus is located at the midsole of the visual cortex (35), fusiform face area. Previous studies have indicated that the fusiform gyrus is involved in the visual cognitive function of facial recognition (36). Face recognition areas have separate brain processing areas, and individuals with agnosia are likely to retain the ability to recognize faces at the same time. The possible explanation may be that their normal object recognition neural pathways is impaired, but the fusiform gyrus face area is not (37). Jiang et al (38) indicated that face classification in visual scenes may begin with the high-order region of the right fusiform gyrus, which is consistent with the observation that the ALFF value is increased in the fusiform gyrus. It may be assumed that blurred vision and ulceration caused by CUs affect facial recognition of patients.

The angular gyrus (ANG) is an important associative region in the back of the brain above the Wernicke region and at the apex occipital lobe (39). In terms of response and perception, attention is sensitive to trial history. Although the basis of the sensory motor interactions remains to be elucidated, from a cognitive and neurologic perspective, converging solid evidence from various methods suggested that the right ANG may be of great importance $(40,41)$.

The superior frontal gyrus (SFG), accounts for one third of the frontal lobe of the human brain, is bounded laterally by the superior frontal sulcus. Studies including fMRI experiments have indicated that the superior frontal gyrus is engaged in self-awareness (42) and laughter (43). In the present study, ALFF values in CU patients were markedly higher in bilateral SFG, which suggested intrinsic brain activation in this area. The ALFF method has been successfully applied in ophthalmological diseases, as outlined in an overview of previous studies provided in Table III, and has a huge development prospect.
Table III. Summary of previous studies on the application of the amplitude of low-frequency fluctuation method in ophthalmological diseases.

\begin{tabular}{llc}
\hline First author (year) & \multicolumn{1}{c}{ Disease } & (Refs.) \\
\hline Guo (2010) & High myopia & $(44)$ \\
Huang (2015) & Glaucoma & $(14)$ \\
Huang (2015) & Optic neuritis & $(13)$ \\
Tan (2016) & Strabismus & $(15)$ \\
Li (2016) & Monocular blindness & $(45)$ \\
Tan (2016) & Open-globe injury & $(46)$ \\
Wang (2017) & Diabetic retinopathy \\
Liang (2016) & Amblyopia & $(47)$ \\
Pan (2018) & Acute eye pain & $(48)$ \\
\hline
\end{tabular}

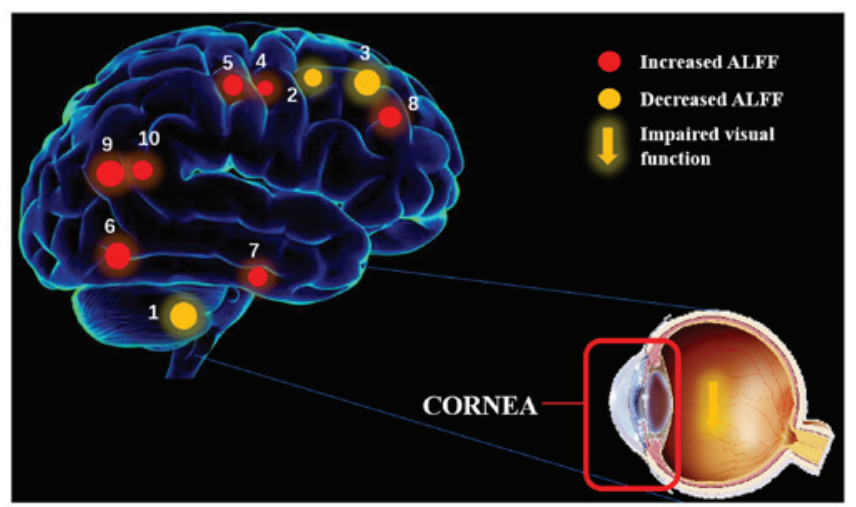

Figure 4. ALFF results of brain activity in the CU group. Compared with the $\mathrm{HCs}$, the ALFF of the following regions was decreased to various extents: 1) Left cerebellar anterior lobe $(\mathrm{t}=-5.35), 2)$ right middle frontal gyrus ( $\mathrm{t}=-4.5573), 3)$ left middle frontal gyrus $(\mathrm{t}=-4.1103)$, 4) right cerebellar inferior lobe $(\mathrm{t}=5.557), 5)$ left cerebellar inferior lobe $(\mathrm{t}=5.786), 6)$ left inferior temporal gyrus $(\mathrm{t}=4.4602), 7)$ right fusiform gyrus $(\mathrm{t}=4.4716), 8)$ left superior frontal gyrus $(\mathrm{t}=4.8905)$, 9) right angular gyrus $(\mathrm{t}=5.2397)$ and 10) bilateral superior frontal gyrus $(\mathrm{t}=4.8908)$. The sizes of the spots denote the degree of quantitative changes. HCs, healthy controls; ALFF, amplitude of low-frequency fluctuation; CU, corneal ulcer.

ROC curve analysis provides a statistical method to distinguish diseased from healthy subjects. The accuracy is considered perfect for AUC values of 0.7-0.9, while values between 0.5 and 0.7 indicate moderate predictability and those $<0.5$ indicate that the discrimination ability is low. The ROC curve analysis of the present study indicated that the AUCs of each brain region were $>0.7$, which suggested that these specific ALFF differences have a proper diagnostic accuracy in characterizing CU. In a word, the present results illustrated that the ALFF method may be a sufficient biomarker of fMRI for the basic research and study of eye diseases. In conclusion, the present study indicates the presence of brain activity disorders in CU patients. These novel results may offer significant information to explain the potential neural effects of CU.

\section{Acknowledgements}

Not applicable. 


\section{Funding}

No funding was received.

\section{Availability of data and materials}

The datasets used and/or analyzed during the current study are available from the corresponding author on reasonable request.

\section{Authors' contributions}

All authors made substantial contributions to this research. WQS, WW and LY performed the experiments and collected the data. WFL, YQS, TS and QL designed the current study. YLM and BL contributed to designing the study and were involved in data interpretation and writing the discussion. PWZ, NJ and YS performed the MRI scanning. WQS wrote the manuscript. All authors have read and approved the final manuscript.

\section{Ethics approval and consent to participate}

The present study was approved by the ethics committee of the First Affiliated Hospital of Nanchang University.

\section{Patient consent for publication}

Not applicable.

\section{Competing interests}

The authors declare that they have no competing interests.

\section{References}

1. Wu J, Zhang WS, Zhao J and Zhou HY: Review of clinical and basic approaches of fungal keratitis. Int J Ophthalmol 9: $1676-1683,2016$

2. World Health Organization: Causes of blindness and visual impairment, 2016.

3. Ngoie Maloba V, Ngayuna Nkiene J, Tunku Kabamba G and Chenge Borasisi G: Frequency of corneal ulcer: Retrospective study of 380 cases carried out in two centers in the DR Congo. J Fr Ophtalmol 41: 57-51, 2018 (In French).

4. El Sheha H: Self-retained amniotic membrane for dendritic keratitis. Ascrs, 2015.

5. Mascarenhas J, Lalitha P, Prajna NV, Srinivasan M, Das M, D'Silva SS, Oldenburg CE, Borkar DS, Esterberg EJ, Lietman TM and Keenan JD: Acanthamoeba, fungal, and bacterial keratitis: A comparison of risk factors and clinical features. Am J Ophthalmol 157: 56-62, 2014.

6. Thomas PA and Kaliamurthy J: Mycotic keratitis: Epidemiology, diagnosis and management. Clin Microbiol Infect 19: 210-220, 2013.

7. Bekiesińskafigatowska M, Helwich E, Rutkowska M, Stankiewicz J and Terczyńska I: Magnetic resonance imaging of neonates in the magnetic resonance compatible incubator. Arch Med Sci 12: 1064-1070, 2016.

8. Liu $\mathrm{H}$ and Wang X: Correlation of iron deposition and change of gliocyte metabolism in the basal ganglia region evaluated using magnetic resonance imaging techniques: An in vivo study. Arch Med Sci 12: 163-171, 2016.

9. Dai XJ, Liu CL, Zhou RL, Gong HH, Wu B, Gao L and Wang YX: Long-term sleep deprivation decreases the default spontaneous activity and connectivity pattern in healthy male subjects: A resting-state fMRI study. Neuropsychiatr Dis Treat 11: 761-772, 2015.

10. Biswal BB: Resting state fMRI: A personal history. Neuroimage 62: 938-944, 2012.
11. Zhang Y, Zhu C, Chen H, Duan X, Lu F, Li M, Liu F, Ma X, Wang Y, Zeng L, et al: Frequency-dependent alterations in the amplitude of low-frequency fluctuations in social anxiety disorder. J Affect Disord 174: 329-335, 2015.

12. Zuo XN, Di Martino A, Kelly C, Shehzad ZE, Gee DG, Klein DF, Castellanos FX, Biswal BB and Milham MP: The oscillating brain: Complex and reliable. Neuroimage 49: 1432-1445, 2010.

13. Huang X, Cai FQ, Hu PH, Zhong YL, Zhang Y, Wei R, Pei CG, Zhou FQ and Shao Y: Disturbed spontaneous brain-activity pattern in patients with optic neuritis using amplitude of low-frequency fluctuation: A functional magnetic resonance imaging study. Neuropsychiatr Dis Treat 11: 3075-3083, 2015.

14. Huang X, Zhong YL, Zeng XJ, Zhou F, Liu XH, Hu PH, Pei CG, Shao Y and Dai XJ: Disturbed spontaneous brain activity pattern in patients with primary angle-closure glaucoma using amplitude of low-frequency fluctuation: A fMRI study. Neuropsychiatr Dis Treat 11: 1877-1883, 2015.

15. Tan G, Huang X, Zhang Y, Wu AH, Zhong YL, Wu K, Zhou FQ and Shao Y: A functional MRI study of altered spontaneous brain activity pattern in patients with congenital comitant strabismus using amplitude of low-frequency fluctuation. Neuropsychiatr Dis Treat 12: 1243-1250, 2016.

16. Satterthwaite TD, Elliott MA, Gerraty RT, Gerraty RT, Ruparel K, Loughead J, Calkins ME, Eickhoff SB, Hakonarson H, Gur RC, Gur RE and Wolf DH: An improved framework for confound regression and filtering for control of motion artifact in the preprocessing of resting-state functional connectivity data. Neuroimage 64: 240-256, 2013.

17. Yan CG, Cheung B, Kelly C, Colcombe S, Craddock RC, Di Martino A, Li Q, Zuo XN, Castellanos FX and Milham MP: A comprehensive assessment of regional variation in the impact of head micromovements on functional connectomics. Neuroimage 76: 183-201, 2013.

18. Fox MD, Snyder AZ, Vincent JL, Corbetta M, Van Essen DC and Raichle ME: The human brain is intrinsically organized into dynamic, anticorrelated functional networks. Proc Natl Acad Sci USA 102: 9673-9678, 2005.

19. Li HJ, Dai XJ, Gong HH, Nie X, Zhang W and Peng DC: Aberrant spontaneous low-frequency brain activity in male patients with severe obstructive sleep apnea revealed by resting-state functional MRI. Neuropsychiatr Dis Treat 11: 207-214, 2015.

20. Dai XJ, Peng DC, Gong HH, Wan AL, Nie X, Li HJ and Wang YX: Altered intrinsic regional brain spontaneous activity and subjective sleep quality in patients with chronic primary insomnia: A restingstate fMRI study. Neuropsychiatr Dis Treat 10: 2163-2175, 2014.

21. Saad ZS, Gotts SJ, Murphy K, Chen G, Jo HJ, Martin A and Cox RW: Trouble at rest: How correlation patterns and group differences become distorted after global signal regression. Brain Connect 2: 25-32, 2012.

22. Zang YF, He Y, Zhu CZ, Cao QJ, Sui MQ, Liang M, Tian LX, Jiang TZ and Wang YF: Altered baseline brain activity in children with ADHD revealed by resting-state functional MRI. Brain Dev 29: 83-91, 2007.

23. Siddharth K, Michael AM, Cahill ND, Kiehl KA, Pearlson G, Baum SA and Calhoun VD: ICA-fNORM: Spatial normalization of fMRI data using intrinsic group-ICA networks. Front Syst Neurosci 5: 93, 2011.

24. Japee S, Holiday K, Satyshur MD, Mukai I and Ungerleider LG: A role of right middle frontal gyrus in reorienting of attention: A case study. Front Syst Neurosci 9: 23, 2015.

25. Morgan HM, Jackson MC, van Koningsbruggen MG, Shapiro KL and Linden DE: Frontal and parietal theta burst TMS impairs working memory for visual-spatial conjunctions. Brain Stimul 6: 122-129, 2013.

26. Chang CC, Yu SC, Mcquoid DR, Messer DF, Taylor WD, Singh K, Boyd BD, Krishnan KR, MacFall JR, Steffens DC and Payne ME: Reduction of dorsolateral prefrontal cortex gray matter in late-life depression. Psychiatry Res 193: 1-6, 2011.

27. Nelson JD, Craig JP, Akpek EK, Azar DT, Belmonte C, Bron AJ, Clayton JA, Dogru M, Dua HS, Foulks GN, et al: TFOS DEWS II introduction. Ocul Surf 15: 269-275, 2017.

28. Leat SJ: A proposed model for integrated low-vision rehabilitation services in Canada. Optom Vis Sci 93: 77-84, 2016.

29. Bruce ML and Hoff RA: Social and physical health risk factors for first-onset major depressive disorder in a community sample. Soc Psychiatry Psychiatr Epidemiol 29: 165-171, 1994.

30. Ferrari C, Oldrati V, Gallucci M, Vecchi T and Cattaneo Z: The role of the cerebellum in explicit and incidental processing of facial emotional expressions: A study with transcranial magnetic stimulation. Neuroimage 169: 256-264, 2018. 
31. Adamaszek M, D'Agata F, Ferrucci R, Habas C, Keulen S, Kirkby KC, Leggio M, Mariën P, Molinari M, Moulton E, et al: Consensus paper: Cerebellum and emotion. Cerebellum 16: 552-576, 2017

32. Kilts CD, Kelsey JE, Knight B, Ely TD, Bowman FD, Gross RE, Selvig A, Gordon A, Newport DJ and Nemeroff CB: The neural correlates of social anxiety disorder and response to pharmacotherapy. Neuropsychopharmacology 31: 2243-2253, 2006.

33. Dien J, Brian ES, Molfese DL and Gold BT: Combined ERP/fMRI evidence for early word recognition effects in the posterior inferior temporal gyrus. Cortex 49: 2307-2321, 2013

34. Stoeter P, Bauermann T, Nickel R, Corluka L, Gawehn J, Vucurevic G, Vossel G and Egle UT: Cerebral activation in patients with somatoform pain disorder exposed to pain and stress: An fMRI study. Neuroimage 36: 418-430, 2007.

35. James CE, Oechslin MS, Van De Ville D, Hauert CA, Descloux C and Lazeyras F: Musical training yields opposite effects on grey matter density in cognitive versus sensorimotor networks. Brain Struct Funct 219: 353-366, 2014.

36. Weiner KS: On (ab)normality: Einstein's fusiform gyrus. Brain Cogn 94: 1-3, 2015.

37. Caspers J, Palomero-Gallagher N, Caspers S, Schleicher A, Amunts K and Zilles K: Receptor architecture of visual areas in the face and word-form recognition region of the posterior fusiform gyrus. Brain Struct Funct 220: 205-219, 2015.

38. Jiang F, Dricot L, Weber J, Righi G, Tarr MJ, Goebel R and Rossion B: Face categorization in visual scenes may start in a higher order area of the right fusiform gyrus: Evidence from dynamic visual stimulation in neuroimaging. J Neurophysiol 106 : 2720-36, 2011

39. Yazar Y, Bergström ZM and Simons JS: Continuous theta burst stimulation of angular gyrus reduces subjective recollection. PLoS One 9: e110414, 2014.

40. Makris N, Preti MG, Wassermann D, Rathi Y, Papadimitriou GM, Yergatian C, Dickerson BC, Shenton ME and Kubicki M: Human middle longitudinal fascicle: Segregation and behavioral-clinical implications of two distinct fiber connections linking temporal pole and superior temporal gyrus with the angular gyrus or superior parietal lobule using multi-tensor tractography. Brain Imaging Behav 7: 335-352, 2013.
41. Bocca F, Töllner T, Müller HJ and Taylor PC: The right angular gyrus combines perceptual and response-related expectancies in visual search: TMS-EEG evidence. Brain Stimul 8: 816-822, 2015.

42. Goldberg I, Harel M and Malach R: When the brain loses its self: Prefrontal inactivation during sensorimotor processing. Neuron 50: 329-339, 2006.

43. Fried I, Wilson C, MacDonald K and Behnke EJ: Electric current stimulates laughter. Nature 391: 650, 1998.

44. Guo MX, Dong HH, Zhang YT, Zhang Q and Yin XH: ALFF changes in brain areas of human with high myopia revealed by resting-state functional MRI. International Conference on Biomedical Engineering and Informatics. IEEE: 91-94, 2010.

45. Li Q, Xin H, Lei Y, Wei R, Zhang Y, Zhong YL, Jiang N and Shao Y: Altered spontaneous brain activity pattern in patients with late monocular blindness in middle-age using amplitude of low-frequency fluctuation: A resting-state functional MRI study. Clin Interv Aging 11: 1773-1780, 2016.

46. Tan G, Huang X, Ye L, Wu AH, He LX, Zhong YL, Jiang N, Zhou FQ and Shao Y: Altered spontaneous brain activity patterns in patients with unilateral acute open globe injury using amplitude of low-frequency fluctuation: A functional magnetic resonance imaging study. Neuropsychiatr Dis Treat 12: 2015-2020, 2016.

47. Wang ZL, Zou L, Lu ZW, Xie XQ, Jia ZZ, Pan CJ, Zhang GX and Ge XM: Abnormal spontaneous brain activity in type 2 diabetic retinopathy revealed by amplitude of low-frequency fluctuations: A resting-state fMRI study. Clin Radiol 72: 340.e1-340.e7, 2017.

48. Liang M, Xie B, Yang H, Yu L, Yin X, Wei L and Wang J: Distinct patterns of spontaneous brain activity between children and adults with anisometropic amblyopia: A resting-state fMRI study. Graefes Arch Clin Exp Ophthalmol 254: 569-576, 2016.

49. Pan ZM, Li HJ, Bao J, Jiang N, Yuan Q, Freeberg S, Zhu PW, Ye L, Ma MY, Huang X and Shao Y: Altered intrinsic brain activities in patients with acute eye pain using amplitude of low-frequency fluctuation: A resting-state fMRI study. Neuropsychiatr Dis Treat 14: 251-257, 2018. 